\title{
Analysis of TAx4 hybrid trigger and events
}

\section{Sang Woo Kim ${ }^{a, *}$ on behalf of the Telescope Array Collaboration}

(a complete list of authors can be found at the end of the proceedings)

${ }^{a}$ Department of Physics, Sungkyunkwan University,

Jang-an-gu, Suwon, Republic of Korea

E-mail: kswowo@naver.com

The Telescope Array is the largest ultra-high energy cosmic ray hybrid detector in the Northern hemisphere. While the TAx4 Surface Detector (TAx4 SD) has a duty cycle of $100 \%$, it should be noted that the TAx4 Fluorescence Detector (TAx4 FD) observes the full longitudinal profile of the cosmic ray air showers and therefore is able to determine their energies more accurately than TAx4 SD. In addition, observing cosmic rays in hybrid mode ("hybrid events") has several advantages. Events seen in hybrid mode by the TAx4 FD and SD are used to establish the energy scale of the TAx4 SD. Moreover, the FD longitudinal profile is used to determine the mass composition of the primary comic ray particles, when the event geometries are well constrained by FD and SD measurements simultaneously. Despite large differences in the TAx4 SD/FD standalone performances, both detector types complement each other in measuring important physical quantities. Since August 2019, direction, energy, and $X_{\max }$ can be obtained from reconstructing hybrid events. In this poster, preliminary analysis of TAx 4 hybrid trigger and TAx 4 hybrid events will be presented with focus on energy and $X_{\max }$ observations.

$37^{\text {th }}$ International Cosmic Ray Conference (ICRC 2021)

July 12 th - 23rd, 2021

Online - Berlin, Germany

\footnotetext{
${ }^{*}$ Presenter
} 


\section{Introduction}

TA [1] found evidence for a cluster of arrival directions of cosmic rays with $\mathrm{E}>57 \mathrm{EeV}$ (hotspot) [2]. To confirm this hotspot including subsequent TA anisotropy results [3, 4], we made a TAx4 plan to quadruple the TA to accelerate the data collection speed. We deployed 257 of the planned 500 plastic scintillation detectors on a square grid with 2.08-km spacing for the TAx 4 SD in February and March of 2019. The total area including the TA SD is approximately 2.5 times larger than the TA SD. The basic design of the TAx4 SD is the same as that of the TA SD. Each surface detector consists of two layers of plastic scintillators, each with one photomultiplier (PMT). Two TAx4 FD stations were constructed, one with four telescopes at the Middle Drum (MD) site and the other with eight telescopes at the Black Rock (BR) site, to confirm the energy scale of cosmic-ray events measured with SD and increase the number of cosmic-ray events for accurate measurement of the depth of air shower maximum $\left(X_{\max }\right)$ related to mass composition utilizing hybrid events simultaneously observed with FD and SD. The TAx4 MD FD has been taking data regularly since June, 2018. The TAx4 BR FD has been taking data regularly since October, 2019. Figure 1 shows the layout of the TAx 4 detector. Figure 2 shows the photograph of the TAx 4 SD deployed in the field on the left panel and the TAx4 MD FD telescopes on the right panel.

\section{Hybrid Trigger}

When a primary ultra-high-energy cosmic ray (UHECR) enters the atmosphere, it generates an extensive air shower. The mass composition of UHECRs can be inferred from the measurement of $X_{\max }$ and provides a strong constraint on models describing UHECR sources and hadron interaction models. UHECR composition can be measured directly up to about $10^{14} \mathrm{eV}$, but the composition around and above $10^{15} \mathrm{eV}$ is indirectly measured using extensive air showers by the comparison with Monte Carlo (MC) simulation with hadron interaction models tuned by the data from particle accelerator experiments.

Current statistics of UHECRs above $10^{19} \mathrm{eV}$ observed with TA is not enough to conclude the composition of real data comparing with MC simulation. Therefore the observation of UHECRs with the TAx4 FD is important to increase the statistics especially above $10^{19} \mathrm{eV}$.

Typical accuracy of the measurement of $X_{\max }$ with monocular FD analysis at $10^{19} \mathrm{eV}$ is $\sim 60$ $\mathrm{g} / \mathrm{cm}^{2}$. If the timing of one SD near the core is used for the shower reconstruction mentioned in Section 3, the shower axis is determined better than the analysis using monocular FD only. The expected accuracy of the $X_{\max }$ measurement is $\sim 20 \mathrm{~g} / \mathrm{cm}^{2}$. Therefore, FD and SD hybrid analysis is very important for $X_{\max }$ analysis. However, the data from three adjacent SDs ${ }^{1}$ are required to trigger showers by SD. Shower trigger efficiency for the TAx4 SD is about $30 \%$ at $10^{19} \mathrm{eV}$ and smaller for lower energies. Therefore we installed the system of hybrid trigger system, which is issued from FD after an FD shower trigger and sent to communications towers to take SD signals with greater than 0.3 single-muon peak independent of SD shower trigger.

The latest hybrid trigger system was developed and used for the TA Low Energy Extension (TALE) detector that observes UHECR with energies from $\sim 10^{16} \mathrm{eV}$ to $\sim 10^{19} \mathrm{eV}$. The average rate

\footnotetext{
${ }^{1}$ The condition of air shower trigger by SD is that there are three adjacent SDs with signals greater than 3 single-muon peaks within $14 \mu \mathrm{sec}$ of one another.
} 


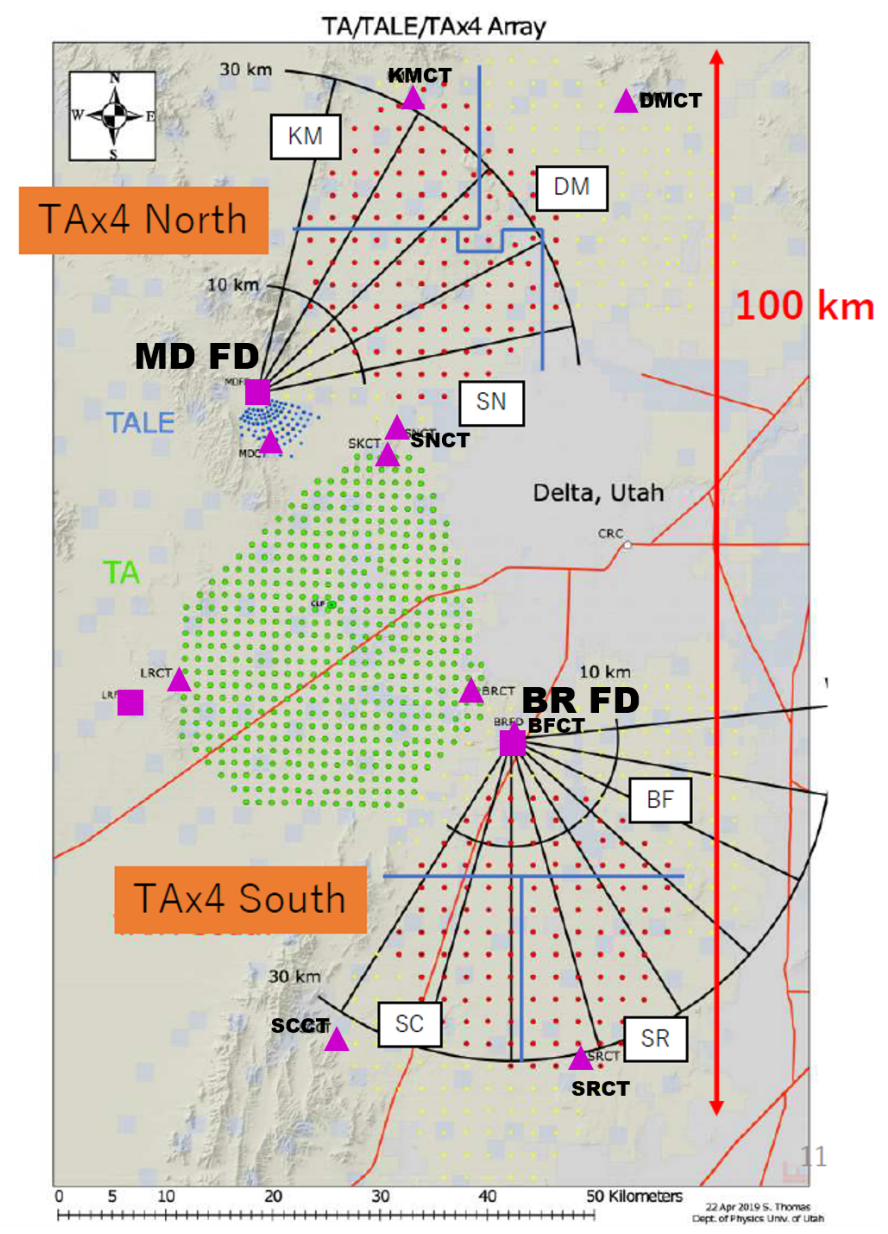

Figure 1: Layout of the TAx4 detector. Red circles denote deployed TAx4 SDs. Yellow dots denote planned TAx4 SDs. Purple triangles denote the communications towers (SNCT, KMCT, and DMCT in the northern lobe; BFCT, SCCT, and SRCT in the southern lobe for TAx4). The TAx4 SD array is divided into six sub-arrays (KM, DM, SN, BF, SC and SR). Blue lines show the boundaries of the TAx4 SD sub-arrays. Green circles denote the TA SDs. Blue circles denote the TALE SDs. Purple squares denote the FD stations (the TA, TAx4 and TALE FDs at the MD site and the TA and TAx4 FDs at the BR site).
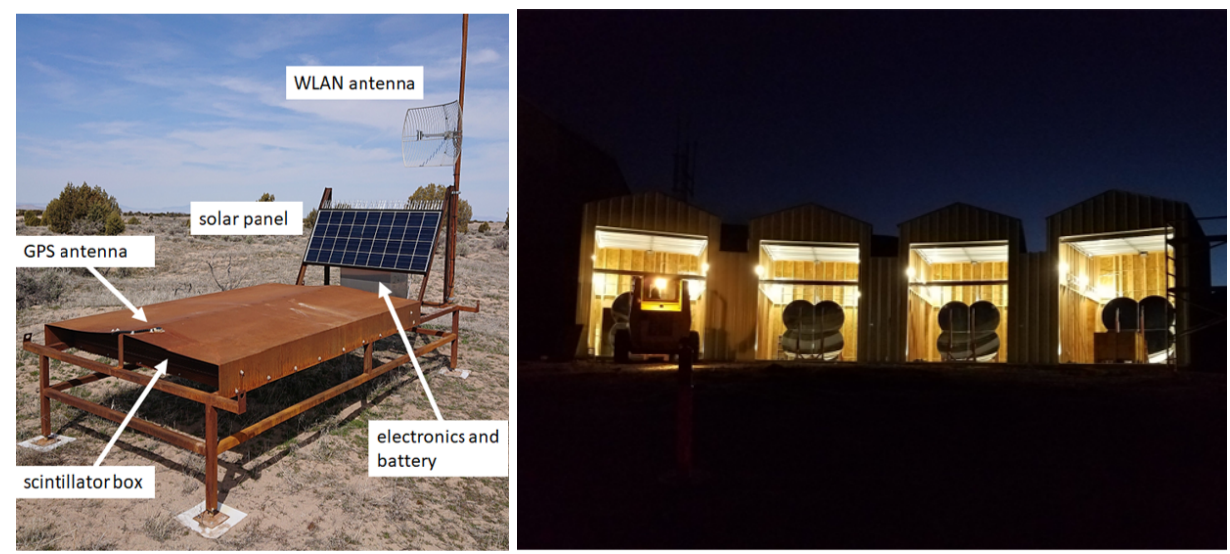

Figure 2: (Left) A TAx4 SD deployed in the field. (Right) The telescopes at the TAx4 MD site. 
of hybrid trigger rate is about $50 \mathrm{mHz}$. We use the same system for TAx4. As a result, we obtained a hybrid trigger rate of about $5 \mathrm{mHz}$ on average and about $15 \mathrm{mHz}$ at maximum.

The scheme of the flow of the hybrid trigger is shown in figure 3 .

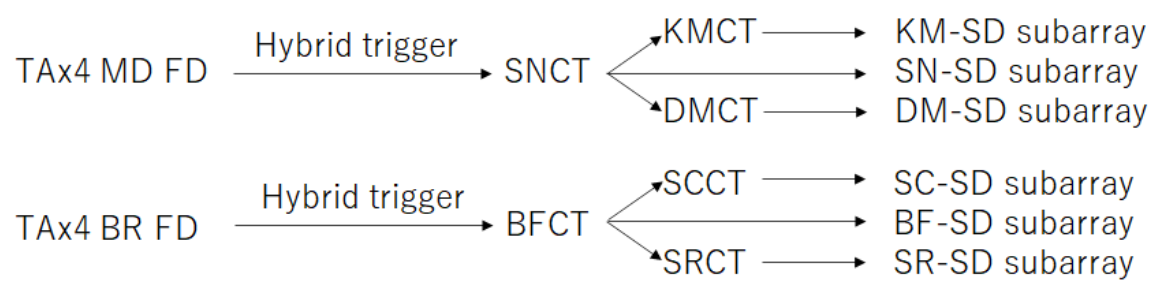

Figure 3: Schematic diagram of the TAx4 hybrid trigger flow. The MD FD and the BR FD are the FD stations at the MD site and the BR site, respectively. The flows from the TAx4 MD FD and the TAx4 BR FD are the systems in the northern lobe and southern lobe, respectively. SNCT, KMCT, DMCT, BFCT, SCCT and SRCT are communications towers explained in figure 1 caption. The corresponding SD subarrays are described as SN-SD, KM-SD, DM-SD, BF-SD, SC-SD and SR-SD subarrays.

\section{Reconstruction Method}

The events that are reconstructed in hybrid-trigger mode have their geometry determined by the following procedure as shown in figure 4. Firstly, the shower detector plane (SDP) is reconstructed from the pattern and pointing direction of the triggered photomultipliers (PMTs). The shower geometry is calculated using the time versus angle fit that uses the pointing directions and timings of the PMTs. The expected timing of $i$-th PMT is described as

$$
t_{\mathrm{exp}, i}=t_{\mathrm{core}}+\frac{1}{c} \frac{\sin \psi-\sin \alpha_{i}}{\sin \left(\psi+\alpha_{i}\right)} r_{\mathrm{core}}
$$

Here $t_{\exp , i}$ and $\alpha_{i}$ are the expected timing and elevation angle in the SDP for the $i$-th PMT, respectively. $t_{\text {core }}$ is the timing when the air shower arrived at the ground. $r_{\text {core }}$ is the distance from the FD station to the shower core. $\psi_{i}$ is an shower inclination angle in the SDP.

For an event that has the timing information of one SD near the shower core, $t_{\text {core }}$ is expressed as

$$
t_{\mathrm{core}}=t_{\mathrm{SD}}+\frac{1}{c}\left(r_{\mathrm{core}}-r_{\mathrm{SD}}\right) \cos \psi
$$

Here $t_{\mathrm{SD}}$ is the timing of the leading edge of the SD signal and $t_{\mathrm{SD}}$ is the distance from the FD station to the SD. Once the shower geometry is determined, the profile of the air shower is fitted using the Gaisser-Hillas parametrization formula,

$$
N(x)=N_{\text {max }}+\left(\frac{x-X_{0}}{X_{\max }-X_{0}}\right)^{\frac{X_{\max }-X_{0}}{\lambda}} \exp \left(\frac{X_{\max }-X_{0}}{\lambda}\right) .
$$

Here $N(x)$ is the number of charged particles at a given slant depth, $x . N_{\max }$ is the maximum number of particles at $X_{\max } . X_{0}$ is the depth of the first interaction and $\lambda$ is the interaction length of shower particles. 


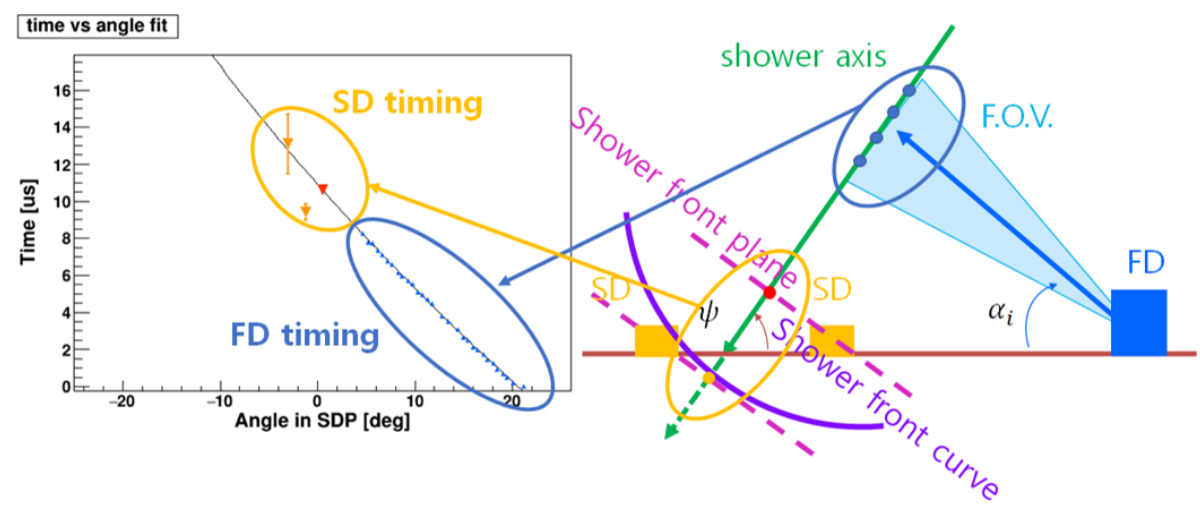

Figure 4: Schematic diagram of the TAx4 hybrid reconstruction. Left) angle in SDP (hybrid). Right) parameters used for reconstruction.

\section{Data}

The TAx4 monocular FD and hybrid analyses are described in [7, 8], and TAx4 SD analyses are described in $[9,10]$. The hybrid trigger system has been operating stably since June 2020. Figure 5 shows the On-time of hybrid-triggers in the northern and southern SD lobes.

To select good example events shown in Section 5, the following quality cuts are applied in the same way as the TA hybrid analysis:

- $500 \mathrm{~g} / \mathrm{cm}^{2}\left(X_{\text {start }}\right)<X_{\max }<1200 \mathrm{~g} / \mathrm{cm}^{2}\left(X_{\text {end }}\right)$,

- $\chi^{2} /$ ndf $<6$,

- $0<\psi<55$ ( $\psi$ : Zenith angle in degrees).

Here $\chi^{2}$ is the $\chi^{2}$ of the geometry fit, and ndf is the number of degrees of freedom.

\section{Results}

Here examples of hybrid-trigger reconstruction for events with hybrid triggers in the northern lobe are shown. Figure 6 is the hybrid reconstruction fit result for a real event that was self-triggered by SD. Table 1 summarizes the reconstructed zenith angle, azimuth angle, core $\mathrm{x}$, core $\mathrm{y}$, energy and $X_{\max }$ obtained by hybrid analysis. Figure 7 is the hybrid reconstruction fit result for a real event that was not self-triggered by SD. Table 2 summarizes the results by hybrid analysis.

\begin{tabular}{|l|r|r|r|r|r|r|}
\hline Analysis & $\begin{array}{r}\text { zenith } \\
(\mathrm{deg})\end{array}$ & $\begin{array}{r}\text { azimuth } \\
(\mathrm{deg})\end{array}$ & $\begin{array}{r}\text { core } \mathrm{x} \\
(\mathrm{km})\end{array}$ & $\begin{array}{r}\text { core y } \\
(\mathrm{km})\end{array}$ & $\begin{array}{r}\log _{10} \mathrm{E} \\
(\mathrm{eV})\end{array}$ & $\begin{array}{r}X_{\max } \\
\left(\mathrm{g} / \mathrm{cm}^{2}\right)\end{array}$ \\
\hline \hline Hybrid & 14.6 & 163.1 & 4.803 & 25.112 & 19.22 & 763.2 \\
\hline
\end{tabular}

Table 1: The result of hybrid reconstruction fit to an event that corresponds to figure 6 for zenith angle, azimuth angle, core x position, core y position, $\log _{10}\left(\right.$ energy) and $X_{\max }$.

The detailed analysis of the real data is ongoing along with the study by MC simulation. 


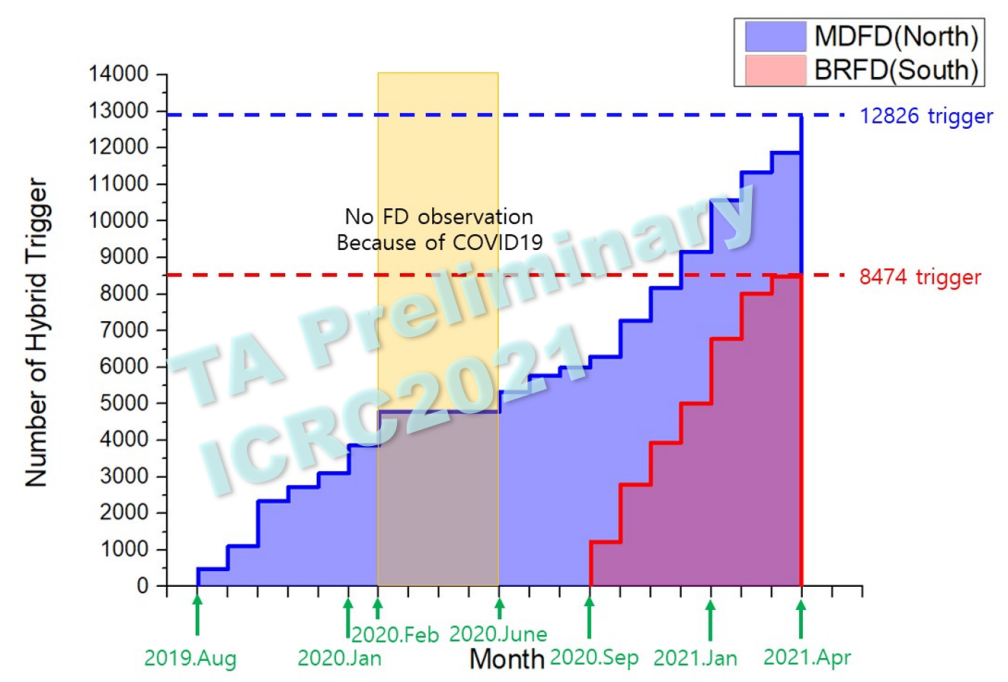

Figure 5: The numbers of TAx4 hybrid triggers per month from the TAx4 MD FD to the TAx4 northern SD subarrays in magenta and from the TAx4 BR FD to the TAx4 southern SD subarrays in pink.

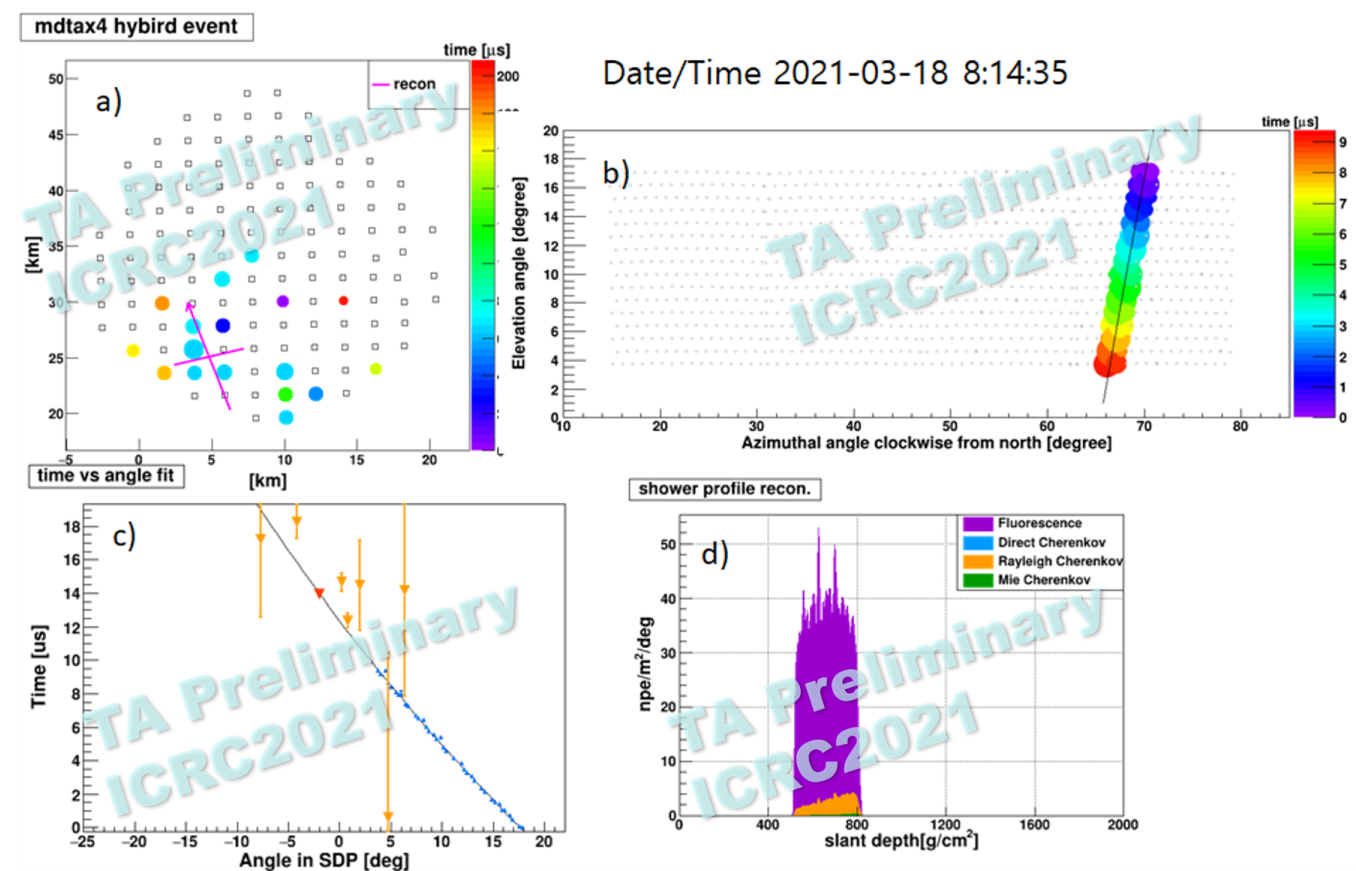

Figure 6: An event recorded at 2021/03/18 08:14:35.588009. (a) SD footprint with shower direction by magenta arrow and shower core position by the cross point. The labels for the $\mathrm{x}$-axis and y-axis denote the position IDs in the west-to-east and the south-to-north directions, respectively.(b) FD display for zenith and azimuth angles. Blue triangles are FD data used for the fit. Inverse triangles are SD data: a red one is used for the fit and others (orange) are not used for the fit. (c) Time versus angle plot using hybrid reconstruction. (d) shower profile. The purple, blue, orange and green areas correspond to fluorescence, direct Cherenkov, Rayleigh Cherenkov and Mie Cherenkov components. 

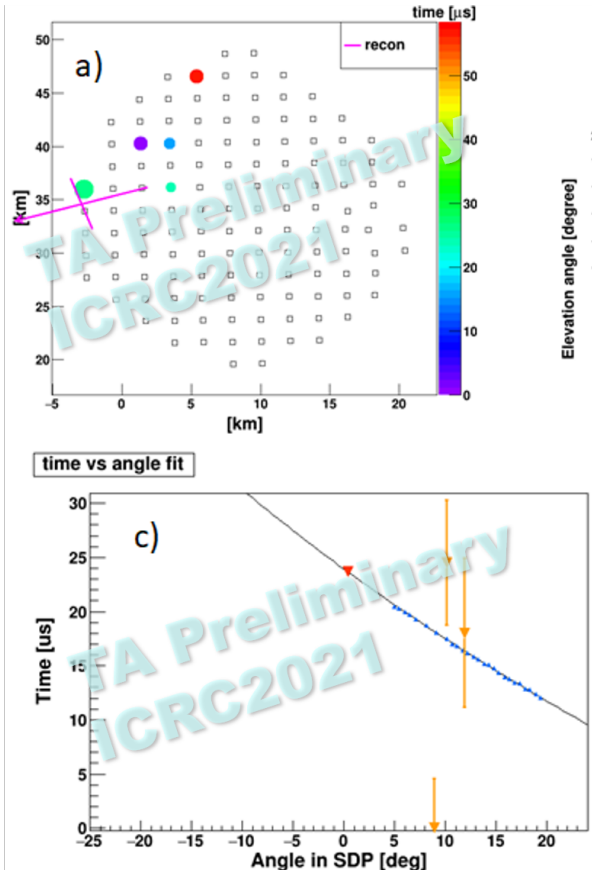

Date/time 2020-11-20 9:14:46
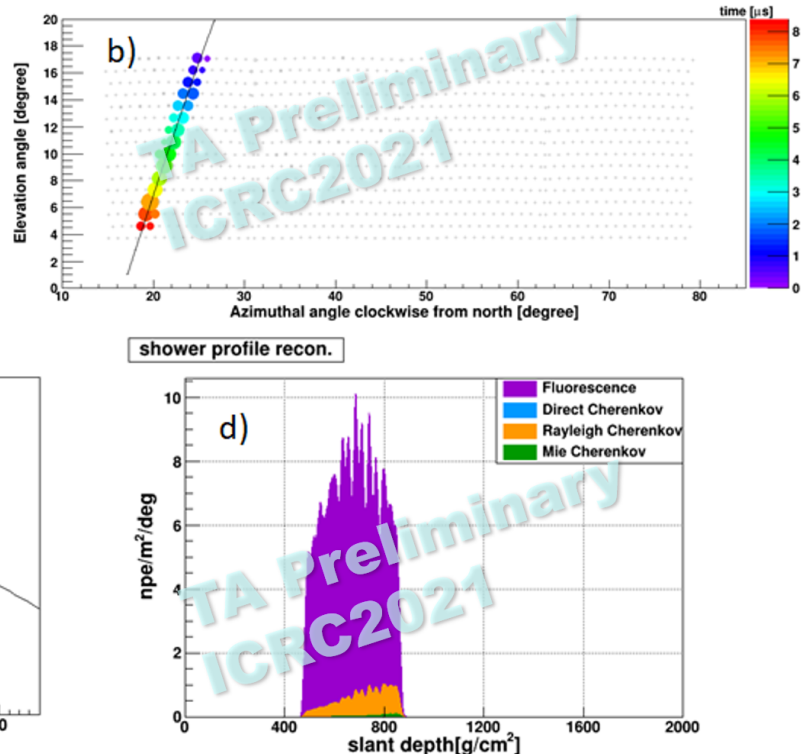

Figure 7: An event recorded at 2020/11/20 09:14:46.663632. (a) SD footprint with shower direction by magenta arrow and shower core position by the cross point. (b) FD display for zenith and azimuth angles. (c) Time versus angle plot using hybrid reconstruction. (d) shower profile.

\begin{tabular}{|r|r|r|r|r|r|r|}
\hline Analysis & $\begin{array}{r}\text { zenith } \\
(\mathrm{deg})\end{array}$ & $\begin{array}{r}\text { azimuth } \\
(\mathrm{deg})\end{array}$ & $\begin{array}{r}\text { core } \mathrm{x} \\
(\mathrm{km})\end{array}$ & $\begin{array}{r}\text { core y } \\
(\mathrm{km})\end{array}$ & $\begin{array}{r}\log _{10} \mathrm{E} \\
(\mathrm{eV})\end{array}$ & $\begin{array}{r}X_{\max } \\
\left(\mathrm{g} / \mathrm{cm}^{2}\right)\end{array}$ \\
\hline \hline Hybrid & 30.2 & 72.0 & -2.914 & 34.567 & 18.73 & 743.3 \\
\hline
\end{tabular}

Table 2: The result of hybrid reconstruction fit to an event that corresponds to figure 7.

\section{Summary}

TA found evidence of anisotropy in the distributions of arrival directions of cosmic rays with energies greater than $57 \mathrm{EeV}$. To collect data more rapidly, we made a TAx4 plan to expand the TA SD array toward four times TA SD adding 500 SDs with 2.08-km spacing. We deployed 257 SDs in February and March of 2019. The currently expanded SD array is approximately 2.5 times larger than the TA SD array.

Two TAx4 FD stations are constructed at the MD and BR FD station sites, respectively, to confirm the energy scale of cosmic-ray events observed with SD and increase the number of cosmicray events with FD for accurate measurement of $X_{\max }$. The TAx4 MD FD and TAx4 BR FD have been taking data regularly since June, 2018 and October, 2019, respectively.

FD and SD hybrid analysis is very important for $X_{\max }$ analysis and energy scale determination. Since the statistics of highest-energy cosmic rays is not enough, it is necessary to comfirm the $X_{\max }$ and energy scale including lower-energy cosmic rays. However, three adjacent SD hits are required to trigger showers with SD. The TAx4 SD shower trigger efficiency is about $30 \%$ at $10^{19} \mathrm{eV}$ and smaller for lower energies. Therefore we installed hybrid trigger system. It is issued from FD and 
sent to communications towers to collect SD hit data with signals greater than 0.3 single-muon peak independent of SD shower trigger. The TAx4 MD and TAx4 BR hybrid trigger systems have been functional stably since August 2019 and September 2020, respectively.

We showed examples of hybrid reconstruction fit to real events, especially for the event without SD self trigger. Further analysis of hybrid-trigger events is on-going.

\section{References}

[1] Kawai, H. et al. (2008). Telescope Array Experiment. Nucl. Phys. B: Proc. Suppl 175-176:221.

[2] Abbasi, R.U. et al. (2014). Indications of intermediate-scale anisotropy of cosmic rays with energy greater than $57 \mathrm{EeV}$ in the northern sky measured with the surface detector of the Telescope Array experiment. Astrophys. J. Lett., 790(2):L21.

[3] Abbasi, R.U. et al. (2018). Evidence of intermediate-scale Energy Spectrum Anisotropy of Cosmic Rays E $\geq 10^{19.2} \mathrm{eV}$ with the Telescope Array Surface Detector. Astrophys. J. 862(2):91.

[4] Abbasi, R.U. et al. (2020). Evidence for a Supergalactic Structure of Magnetic Deflection Multiplets of Ultra-high-energy Cosmic Rays. Astrophys. J. 899(1):86.

[5] Abbasi, R.U. et al. (2021). Surface detectors of the TAx4 experiment. submitted to Nucl. Instrum. Meth. A.

[6] Abu-Zayyad, T. et al. (2012). The surface detector array of the Telescope Array experiment. Nucl. Instrum. Meth. A, 689:87.

[7] M. Potts et al. Update on the TAx4 Fluorescence Detectors. This conference.

[8] R. Gonzalez et al. TAx4 Hybrid Simulation and Reconstruction. This conference.

[9] H. Jeong et al. Reconstruction of air shower events measured by the surface detectors of the TAx4 experiment. This conference.

[10] K. Fujisue et al. Performance and simulation of the surface detector array of the TAx4 experiment. This conference. 


\section{Full Authors List: Telescope Array Collaboration}

R.U. Abbasi ${ }^{1,2}$, T. Abu-Zayyad ${ }^{1,2}$, M. Allen ${ }^{2}$, Y. Arai ${ }^{3}$, R. Arimura ${ }^{3}$, E. Barcikowski ${ }^{2}$, J.W. Belz ${ }^{2}$, D.R. Bergman ${ }^{2}$, S.A. Blake ${ }^{2}$, I. Buckland ${ }^{2}$, R. $\mathrm{Cady}^{2}$, B.G. Cheon ${ }^{4}$, J. Chiba ${ }^{5}$, M. Chikawa ${ }^{6}$, T. Fujii ${ }^{7}$, K. Fujisue ${ }^{6}$, K. Fujita ${ }^{3}$, R. Fujiwara ${ }^{3}$, M. Fukushima ${ }^{6}$, R. Fukushima ${ }^{3}$, G. Furlich ${ }^{2}$, R. Gonzalez ${ }^{2}$, W. Hanlon ${ }^{2}$, M. Hayashi ${ }^{8}$, N. Hayashida ${ }^{9}$, K. Hibino ${ }^{9}$, R. Higuchi ${ }^{6}$, K. Honda ${ }^{10}$, D. Ikeda ${ }^{9}$, T. Inadomi ${ }^{11}$, N. Inoue ${ }^{12}$, T. Ishii ${ }^{10}$, H. Ito $^{13}$, D. Ivanov ${ }^{2}$, H. Iwakura ${ }^{11}$, A. Iwasaki ${ }^{3}$, H.M. Jeong ${ }^{14}$, S. Jeong ${ }^{14}$, C.C.H. Jui ${ }^{2}$, K. Kadota ${ }^{15}$, F. Kakimoto ${ }^{9}$, O. Kalashev ${ }^{16}$, K. Kasahara ${ }^{17}$, S. Kasami ${ }^{18}$, H. Kawai ${ }^{19}$, S. Kawakami ${ }^{3}$, S. Kawana ${ }^{12}$, K. Kawata ${ }^{6}$, I. Kharuk ${ }^{16}$, E. Kido ${ }^{13}$, H.B. Kim ${ }^{4}$, J.H. Kim ${ }^{2}$, J.H. Kim ${ }^{2}$, M.H. Kim ${ }^{14}$, S.W. Kim ${ }^{14}$, Y. Kimura ${ }^{3}$, S. Kishigami ${ }^{3}$, Y. Kubota ${ }^{11}$, S. Kurisu ${ }^{11}$, V. Kuzmin ${ }^{16}$, M. Kuznetsov ${ }^{16,20}$, Y.J. Kwon ${ }^{21}$, K.H. Lee ${ }^{14}$, B. Lubsandorzhiev ${ }^{16}$, J.P. Lundquist ${ }^{2,22}$, K. Machida ${ }^{10}$, H. Matsumiya ${ }^{3}$, T. Matsuyama ${ }^{3}$, J.N. Matthews ${ }^{2}$, R. Mayta ${ }^{3}$, M. Minamino ${ }^{3}$, K. Mukai ${ }^{10}$, I. Myers ${ }^{2}$, S. Nagataki ${ }^{13}$, K. Nakai ${ }^{3}$, R. Nakamura ${ }^{11}$, T. Nakamura ${ }^{23}$, T. Nakamura ${ }^{11}$, Y. Nakamura ${ }^{11}$, A. Nakazawa ${ }^{11}$, E. Nishio ${ }^{18}$, T. Nonaka ${ }^{6}$, H. Oda $^{3}$, S. Ogio $^{3,24}$, M. Ohnishi ${ }^{6}$, H. Ohoka ${ }^{6}$, Y. Oku ${ }^{18}$, T. Okuda ${ }^{25}$, Y. Omura ${ }^{3}$, M. Ono ${ }^{13}$, R. Onogi ${ }^{3}$, A. Oshima ${ }^{3}$, S. Ozawa ${ }^{26}$, I.H. Park ${ }^{14}$, M. Potts ${ }^{2}$, M.S. Pshirkov ${ }^{16,27}$, J. Remington ${ }^{2}$, D.C. Rodriguez ${ }^{2}$, G.I. Rubtsov ${ }^{16}$, D. Ryu ${ }^{28}$, H. Sagawa ${ }^{6}$, R. Sahara ${ }^{3}$, Y. Saito ${ }^{11}$, N. Sakaki ${ }^{6}$, T. Sako ${ }^{6}$, N. Sakurai ${ }^{3}$, K. Sano ${ }^{11}$, K. Sato ${ }^{3}$, T. Seki ${ }^{11}$, K. Sekino ${ }^{6}$, P.D. Shah ${ }^{2}$, Y. Shibasaki ${ }^{11}$, F. Shibata ${ }^{10}$, N. Shibata ${ }^{18}$, T. Shibata ${ }^{6}$, H. Shimodaira ${ }^{6}$, B.K. Shin ${ }^{28}$, H.S. Shin ${ }^{6}$, D. Shinto ${ }^{18}$, J.D. Smith ${ }^{2}$, P. Sokolsky ${ }^{2}$, N. Sone ${ }^{11}$, B.T. Stokes ${ }^{2}$, T.A. Stroman ${ }^{2}$, Y. Takagi ${ }^{3}$, Y. Takahashi ${ }^{3}$, M. Takamura $^{5}$, M. Takeda ${ }^{6}$, R. Takeishi ${ }^{6}$, A. Taketa $^{29}$, M. Takita ${ }^{6}$, Y. Tameda ${ }^{18}$, H. Tanaka ${ }^{3}$, K. Tanaka ${ }^{30}$, M. Tanaka ${ }^{31}$, Y. Tanoue ${ }^{3}$, S.B. Thomas ${ }^{2}$, G.B. Thomson ${ }^{2}$, P. Tinyakov ${ }^{16,20}{ }^{\text {I. } \text { Tkachev }^{16}, \text { H. Tokuno }}{ }^{32}$, T. Tomida ${ }^{11}$, S. Troitsky ${ }^{16}$, R. Tsuda ${ }^{3}$, Y. Tsunesada ${ }^{3,24}$, Y. Uchihori ${ }^{33}$, S. Udo ${ }^{9}$, T. Uehama ${ }^{11}$, F. Urban ${ }^{34}$, T. Wong ${ }^{2}$, K. Yada $^{6}$, M. Yamamoto ${ }^{11}$, K. Yamazaki ${ }^{9}$, J. Yang ${ }^{35}$, K. Yashiro ${ }^{5}$, F. Yoshida ${ }^{18}$, Y. Yoshioka ${ }^{11}$, Y. Zhezher ${ }^{6,16}$, and Z. Zundel ${ }^{2}$

${ }^{1}$ Department of Physics, Loyola University Chicago, Chicago, Illinois, USA

${ }^{2}$ High Energy Astrophysics Institute and Department of Physics and Astronomy, University of Utah, Salt Lake City, Utah, USA

${ }^{3}$ Graduate School of Science, Osaka City University, Osaka, Osaka, Japan

${ }^{4}$ Department of Physics and The Research Institute of Natural Science, Hanyang University, Seongdong-gu, Seoul, Korea

${ }^{5}$ Department of Physics, Tokyo University of Science, Noda, Chiba, Japan

${ }^{6}$ Institute for Cosmic Ray Research, University of Tokyo, Kashiwa, Chiba, Japan

7 The Hakubi Center for Advanced Research and Graduate School of Science, Kyoto University, Kitashirakawa-Oiwakecho, Sakyo-ku, Kyoto, Japan

${ }^{8}$ Information Engineering Graduate School of Science and Technology, Shinshu University, Nagano, Nagano, Japan

${ }^{9}$ Faculty of Engineering, Kanagawa University, Yokohama, Kanagawa, Japan

${ }^{10}$ Interdisciplinary Graduate School of Medicine and Engineering, University of Yamanashi, Kofu, Yamanashi, Japan

${ }^{11}$ Academic Assembly School of Science and Technology Institute of Engineering, Shinshu University, Nagano, Nagano, Japan

12 The Graduate School of Science and Engineering, Saitama University, Saitama, Saitama, Japan 
${ }^{13}$ Astrophysical Big Bang Laboratory, RIKEN, Wako, Saitama, Japan

${ }^{14}$ Department of Physics, SungKyunKwan University, Jang-an-gu, Suwon, Korea

${ }^{15}$ Department of Physics, Tokyo City University, Setagaya-ku, Tokyo, Japan

${ }^{16}$ Institute for Nuclear Research of the Russian Academy of Sciences, Moscow, Russia

${ }^{17}$ Faculty of Systems Engineering and Science, Shibaura Institute of Technology, Minato-ku, Tokyo, Japan

18 Department of Engineering Science, Faculty of Engineering, Osaka Electro-Communication University, Neyagawa-shi, Osaka, Japan

${ }^{19}$ Department of Physics, Chiba University, Chiba, Chiba, Japan

${ }^{20}$ Service de Physique Théorique, Université Libre de Bruxelles, Brussels, Belgium

${ }^{21}$ Department of Physics, Yonsei University, Seodaemun-gu, Seoul, Korea

${ }^{22}$ Center for Astrophysics and Cosmology, University of Nova Gorica, Nova Gorica, Slovenia

${ }^{23}$ Faculty of Science, Kochi University, Kochi, Kochi, Japan

${ }^{24}$ Nambu Yoichiro Institute of Theoretical and Experimental Physics, Osaka City University, Osaka, Osaka, Japan

${ }^{25}$ Department of Physical Sciences, Ritsumeikan University, Kusatsu, Shiga, Japan

${ }^{26}$ Quantum ICT Advanced Development Center, National Institute for Information and Communications Technology, Koganei, Tokyo, Japan

${ }^{27}$ Sternberg Astronomical Institute, Moscow M.V. Lomonosov State University, Moscow, Russia

28 Department of Physics, School of Natural Sciences, Ulsan National Institute of Science and Technology, UNIST-gil, Ulsan, Korea

${ }^{29}$ Earthquake Research Institute, University of Tokyo, Bunkyo-ku, Tokyo, Japan

${ }^{30}$ Graduate School of Information Sciences, Hiroshima City University, Hiroshima, Hiroshima, Japan

${ }^{31}$ Institute of Particle and Nuclear Studies, KEK, Tsukuba, Ibaraki, Japan

${ }^{32}$ Graduate School of Science and Engineering, Tokyo Institute of Technology, Meguro, Tokyo, Japan

${ }^{33}$ Department of Research Planning and Promotion, Quantum Medical Science Directorate, National Institutes for Quantum and Radiological Science and Technology, Chiba, Chiba, Japan

${ }^{34}$ CEICO, Institute of Physics, Czech Academy of Sciences, Prague, Czech Republic

${ }^{35}$ Department of Physics and Institute for the Early Universe, Ewha Womans University, Seodaaemungu, Seoul, Korea

\section{Acknowledgements:}

The Telescope Array experiment is supported by the Japan Society for the Promotion of Science(JSPS) through Grants-in-Aid for Priority Area 431, for Specially Promoted Research JP21000002, for Scientific Research (S) JP19104006, for Specially Promoted Research JP15H05693, for Scientific Research (S) JP15H05741 and JP19H05607, for Science Research (A) JP18H03705, for Young Scientists (A) JPH26707011, and for Fostering Joint International Research (B) JP19KK0074, by the joint research program of the Institute for Cosmic Ray Research (ICRR), The University of Tokyo; by the Pioneering Program of RIKEN for the Evolution of Matter in the Universe (r-EMU); by the U.S. National Science Foundation awards PHY-1404495, PHY-1404502, PHY-1607727, 
PHY-1712517, PHY-1806797 and PHY-2012934; by the National Research Foundation of Korea (2017K1A4A3015188, 2020R1A2C1008230, \& 2020R1A2C2102800) ; by the Ministry of Science and Higher Education of the Russian Federation under the contract 075-15-2020-778, RFBR grant 20-02-00625a (INR), IISN project No. 4.4501.18, and Belgian Science Policy under IUAP VII/37 (ULB). This work was partially supported by the grants ofThe joint research program of the Institute for Space-Earth Environmental Research, Nagoya University and Inter-University Research Program of the Institute for Cosmic Ray Research of University of Tokyo. The foundations of Dr. Ezekiel R. and Edna Wattis Dumke, Willard L. Eccles, and George S. and Dolores Doré Eccles all helped with generous donations. The State of Utah supported the project through its Economic Development Board, and the University of Utah through the Office of the Vice President for Research. The experimental site became available through the cooperation of the Utah School and Institutional Trust Lands Administration (SITLA), U.S. Bureau of Land Management (BLM), and the U.S. Air Force. We appreciate the assistance of the State of Utah and Fillmore offices of the BLM in crafting the Plan of Development for the site. Patrick A. Shea assisted the collaboration with valuable advice and supported the collaboration's efforts. The people and the officials of Millard County, Utah have been a source of steadfast and warm support for our work which we greatly appreciate. We are indebted to the Millard County Road Department for their efforts to maintain and clear the roads which get us to our sites. We gratefully acknowledge the contribution from the technical staffs of our home institutions. An allocation of computer time from the Center for High Performance Computing at the University of Utah is gratefully acknowledged. 